INFLATION IN THE UNIVERSE, CIRCA 1986*

Michael S. Turner

NASA/Fermilab Astrophysics Center

Fermi National Accelerator Laboratory

Batavia, IL 60510

and

Departments of Physics and Astronomy and Astrophysics

Enrico Fermi Institute

The University of Chicago

Chicago, IL 60637

*To appear in the Proceedings of GR 11, held in Stockholm, Sweden, July 1986. 


\title{
INFLATION IN THE UNIVERSE, CIRCA 1986
}

\section{Michael S. Turner}

Fermilab Astrophysics, Fermi National Accelerator Laboratory, Batavia, IL 60510 USA, and Departments of Physics and Astronomy and Astrophysics, Enrico Fermi Insitiute, The University of Chicago, Chicago, IL 60637 USA

\begin{abstract}
The hot big bang cosmology, or the standard cosmology as it is appropriately known, is a highly successful model, providing a reliable and tested accounting of the Universe from $0.01 \mathrm{sec}$ after the bang until today, some $15 \mathrm{Gyr}$ later. However, very special initial data seem to be required in order to account for the observed smoothness and flatness of our Hubble volume and for the existence of the small primeval density inhomogeneities required for the formation of structure in the Universe. Inflation offers a means of accounting for these special initial data which is based upon physics at sub-planck energy scales $\left(\ll m_{p l} \simeq 10^{19} \mathrm{GeV}\right)$ and is motivated by contemporary ideas in particle theory. Here I review the status of the 'Inflationary Paradigm,' Circa 1986. At present essentially all inflationary models involve a very weakly-coupled (quantified by the presence of a dimensionless parameter of order $10^{-12}$ or so) scalar field which is displaced from the minimum of its potential. Regions of the Universe where the scalar field is initially displaced from its minimum undergo inflation as the scalar field relaxes, resulting in a Universe today which resembles ours in regions much larger than our present Hubble volume $\left(\simeq 10^{28} \mathrm{~cm}\right)$, but which on very large scales $\left(\gg 10^{28} \mathrm{~cm}\right)$ may be highly irregular. At present, the most conspicuous blemish on the paradigm is the lack of a compelling particle physics model to implement it. I also briefly review some other unresolved issues facing inflation, including the confrontation between inflation and observational data.
\end{abstract}

\section{SUCCESSES OF THE STANDARD COSMOLOGY}

The standard cosmology is a remarkable achievement. Based upon the Friedmann-Robertson-Walker (FRW) homogeneous and isotropic cosmological model, it provides us with an accurate description of the evolution of the Universe from about $10^{-2} \mathrm{sec}$ after the bang (when the temperature of the Universe was about $10 \mathrm{MeV}$ ) until the present some $3 \times 10^{17} \mathrm{sec}$ later (and temperature $2.75 \mathrm{~K}$ ). Support for the standard cosmology is based upon a triad of observations. First, the isotropic Hubble flow and homogeneous distribution of galaxies; light from the most distant galaxies and QSO's (redshifts of order 3-4) left these objects only a few billion years after the bang and therefore test the model to within a few billion years of the bang. Second, there is the cosmic microwave background radiation (CMBR) whose spectrum is consistent with that of a black body at a temperature of $2.75 \pm 0.05 K$ (Smoot et 
al. 1985; Peterson et al. 1985) and which is spatially uniform to about a part in $10^{4}$ on angular scales from a few arcminutes to 180 degrees (Wilkinson 1986). [The only anisotropy unambiguously detected thus far is the dipole component whose magnitude is of order $10^{-3}$ and whose simplest interpretation is being due to our motion with respect to the cosmic rest frame.] The surface of last scattering for the CMBR is the Universe at $t \simeq 10^{13} \mathrm{sec}$ and $T \simeq 1 / 3 \mathrm{eV}$, and so it provides a probe of the standard model to within a few 100,000 yrs of the bang. Finally, there is the concordance of the cosmic abundances of $\mathrm{D},{ }^{3} \mathrm{He},{ }^{4} \mathrm{He}$, and ${ }^{7} \mathrm{Li}$ with the predictions of big bang nucleosynthesis, providing that the present baryon-to-photon ratio $\eta \simeq(4-7) \times 10^{10}$ (equivalently, $0.014 \lesssim \Omega_{B} h^{2} \lesssim 0.035$, where as usual $H_{0}=100 h \mathrm{kmsec}^{-1} \mathrm{Mpc}^{-1}$ ) (Yang et al. 1984; Boesgaard \& Steigman 1985). According to the standard cosmology there was an epoch of primordial nucleosynthesis from about $t \simeq 0.01 \mathrm{sec}-300 \mathrm{sec}$ $(T \simeq 10 \mathrm{MeV}-0.1 \mathrm{MeV})$, and so the cosmic abundances of these light elements serve to test the model at times to within a fraction of a second after the bang. In addition, the standard cosmology provides a general framework for understanding how structure in the Universe evolved (see, e.g., Efstathiou \& Silk 1983): once the Universe became matter-dominated $\left(t \simeq 10^{10} \mathrm{sec}\right.$ and $\left.T \simeq 10 \mathrm{eV}\right)$, small $\left(\simeq 10^{-4}\right)$ primeval density inhomogeneities grew via the Jeans (or gravitational) instability into the plethora of structure we observe today (galaxies, clusters of galaxies, superclusters, voids, etc.).

During its early history $\left(t \lesssim 10^{10} \mathrm{sec}\right)$ the energy density of the Universe was dominated by relativistic particle species in thermal equilibrium with a temperature $T \simeq(t / s e c)^{-1 / 2} \mathrm{MeV}$, and the cosmic scale factor $R(t) \propto t^{1 / 2}$. While the standard cosmology is only tested back to times of order $10^{-2} \mathrm{sec}$, the standard model of particle physics, the $S U(3)_{c} \otimes S U(2)_{L} \otimes U(1)_{Y}$ gauge theory of the strong, weak, and electromagnetic interactions (believed to be valid at energies $\lesssim 1000 \mathrm{GeV}$ ), and theoretical speculations about physics at very high $\left(\gtrsim 10^{14} \mathrm{GeV}\right.$ ) energies (e.g., grand unification, supersymmetry/supergravity, and superstring theories) allow us to extrapolate the model back to times as early as $10^{-43} \mathrm{sec}$ and perhaps even earlier (see Fig. 1). [At times earlier than $10^{-43} \mathrm{sec}$ (corresponding to temperatures $\gtrsim 10^{19} \mathrm{GeV}$ ) quantum gravitational effects should become very important and extrapolation to times this early necessarily requires a quantum description of gravity.] The speculations have proven very interesting, from extra dimensions, to baryogenesis, to monopoles, to cosmic strings, to relic WIMPs, to phase transitions, and finally inflation, the subject of my talk. Of course, all of these interesting speculations could prove to be nothing more than that; however, nothing in our present knowledge of physics would tell us that such speculations are a priori wrong. Compare this to the situation some 20 years ago when it was thought that hadrons were fundamental: at times earlier than about $10^{-5} \sec$ after the bang inter-particle distances should have been less than the size of a typical hadron, thus precluding sensible speculations about times earlier than this.

\section{SHORTCOMINGS OF THE STANDARD MODEL}

As successful as it is, the standard model has its shortcomings. They 


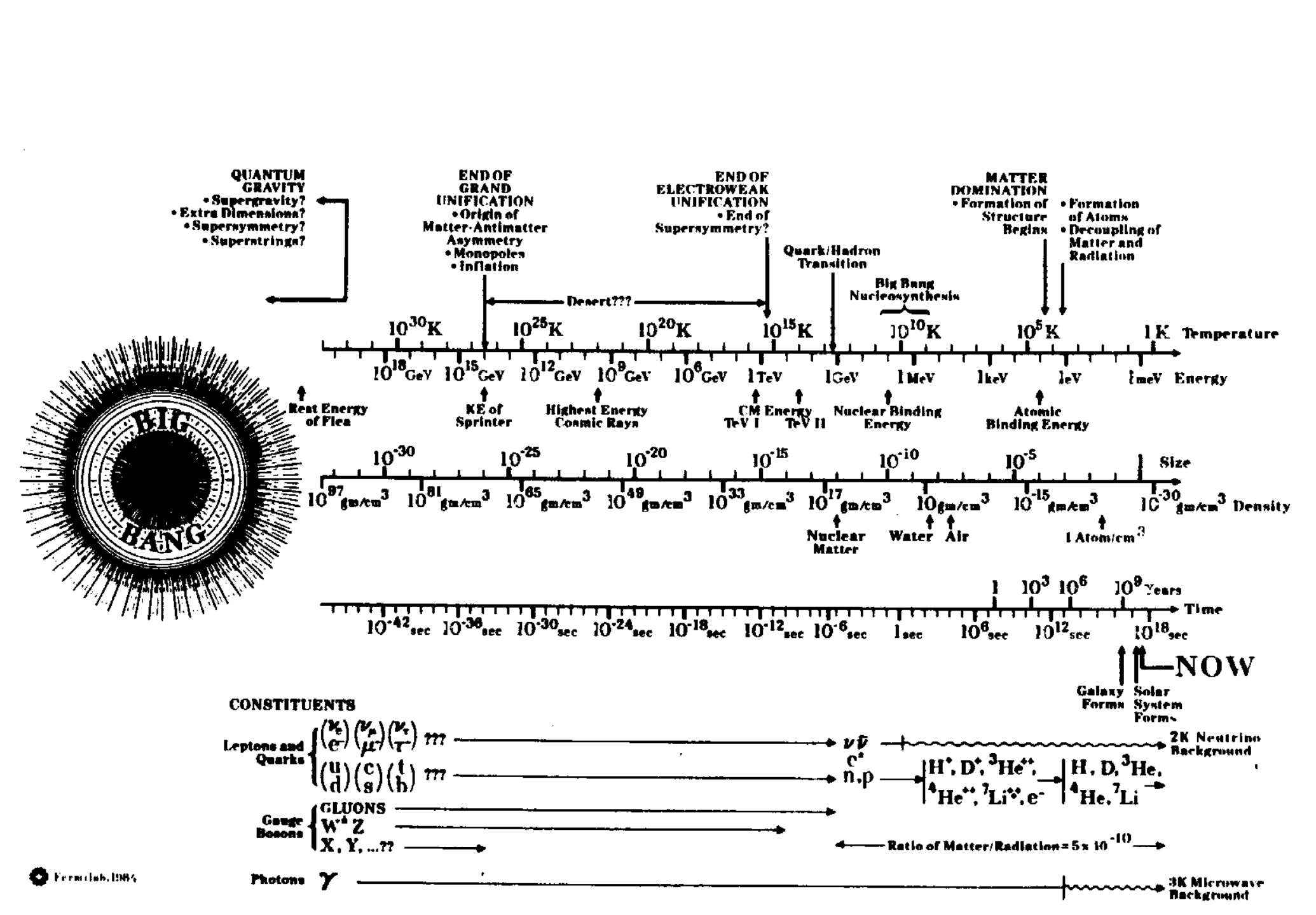


involve a number of very fundamental facts about the Universe we observe within our Hubble volume, which it can accommodate, but by no means provides fundamental explanations for (in contrast, the standard cosmology provides a fundamental explanation for the abundance of the light elements). These cosmological conundrums are by this time very well known; they include: (1) The smoothness (isotropy and homogeneity) of our present Hubble volume (radius $\simeq H^{-1} \simeq 10^{28} \mathrm{~cm}$ ) on scales $\gg 10 M p c$, as evidenced by the uniformity of the CMBR and of the distribution of galaxies. The size of our Hubble volume is conveniently quantified by the entropy within it, which is dominated by the relic photon and neutrino seas and is of order $10^{88}$. Because of the existence of particle horizons in the standard cosmology it is essentially impossible to account for a smooth volume this large as having evolved due to physical processes operating in the early Universe: when matter and radiation last interacted, the Hubble volume at the time contained an entropy of only about $10^{82}$, so that particle interactions could not account for such a large smooth volume. (2) The origin and nature of the primeval fluctuations required to explain the rich array of structure in the Universe today; curvature fluctuations of order $10^{-4}$ on mass scales $\sim 10^{8} M_{\odot}-10^{15} M_{\odot}$ are required. In the standard cosmology, curvature fluctuations cannot arise spontaneously (again because of the existence of particle horizons; see, e.g., Bardeen 1980) and must be put in ab initio. [It is possible that the requisite fluctuations are isocurvature fluctuations and were created during the early history of the Universe, perhaps during a phase transition; a promising example which has attracted a great deal of attention lately is cosmic strings (see, e.g., Vilenkin 1985; Albrecht \& Turok 1985; Turok 1985).] (3) The apparent flatness of our Hubble volume; the radius of curvature $\left(R_{c u r v} \equiv R(t)|k|^{-1 / 2}=H^{-1} /|\Omega-1|^{1 / 2}\right)$ in our vicinity must be at least comparable to the radius of our Hubble volume. Had the radius of curvature been of order the planck length $\left(\simeq 10^{-33} \mathrm{~cm}\right)$ at the planck time $\left(\simeq 10^{-43} \mathrm{sec}\right)$, it would only be of order $0.1 \mathrm{~cm}$ when the Universe reached a temperature of $3 K$. [Put another way, in order that $\Omega$ still not be too different from unity, at the planck time it must have been equal to 1 to within a part in $10^{60}$.] (This dilemma and the naturalness of the flat, Einstein-deSitter model has been emphasized by Dicke \& Peebles 1979.) (4) The net baryon number within our Hubble volume, quantified as the baryon-to-entropy ratio, $n_{B} / s \simeq \eta / 7 \simeq(7-10) \times 10^{-11}$. Of course one of the great successes of the InnerSpace/OuterSpace connection is baryogenesis, the modern theory of the origin of the baryon asymmetry, and it appears that now we at least have a framework for understanding the origin of this very fundamental quantity (for a review see Kolb \& Turner 1983). (5) The dearth (thank goodness) of monopoles and other topological beasts which would have been produced in great excess during the earliest moments of the Universe $\left(t \lesssim 10^{-34} \mathrm{sec}\right)$ had the standard model been valid and if the interactions of nature are unified by a semi-simple gauge group, such as $S U_{5}, S O_{10}$, or $E_{6}$ (for a review see Preskill 1984). (6) The smallness of the present cosmological term. With the possible exception of supersymmetry, no symmetry forbids such a term in the Einstein-Hilbert action, and so on dimensional grounds one would expect 
such a term to be of order $m_{p l}^{2}$, corresponding to a vacuum energy of order $m_{p l}^{4}$. In any case, contributions to the vacuum energy of order $M^{4}$ arise due to spontaneous symmetry breaking (SSB) at the energy scale $M$. The measured expansion rate of the Universe restricts the present vacuum energy contribution to be $\lesssim 10^{-46} \mathrm{GeV}^{4}$. Even the contribution from chiral symmetry breaking ( $M \simeq f e w 100 \mathrm{MeV})-\mathrm{a}$ phenomenon that particle physicists think they know something about, violates this bound by some 42 orders-of-magnitude!

All of these cosmological facts can be accommodated by the standard model, but seemingly at the expense of highly special initial data (the possible exception being the monopole problem). In 1973, Collins and Hawking pointed out that the set of initial data which evolve to a Universe such as ours is of measure zero providing that the stress energy in the Universe has always satisfied the strong and dominant energy conditions. Over the years there have been a number of attempts to try to understand and/or explain this apparent dilemma of initial data. Inflation is the most recent attempt and I believe shows great promise. Let me begin by briefly mentioning the earlier attempts:

* Mixmaster Paradigm-Starting with a solution with a singularity which exhibits the features of the most general singular solutions known (the so-called mixmaster model) Misner and his coworkers hoped that they could show that particle viscosity would smooth out the geometry. In part because horizons still effectively exist in the mixmaster solution this program has proven unsuccessful (see, e.g., Misner 1968, 1972; Matzner \& Misner 1972).

* Nature of the Initial Singularity-Penrose (1979) explored the possibility of explaining the observed smoothness of the Universe by restricting the kinds of initial singularities which are permitted in Nature (those with vanishing Weyl curvature). In a sense his approach is to postulate a law of physics governing allowed initial data.

* Quantum Gravity Effects-The first two solutions involve appealing to classical gravitational effects. A number of authors have suggested that quantum gravity effects might be responsible for smoothing out the space-time geometry (deWitt 1953; Parker 1966; Zel'dovich 1970; Starobinskii 1980; Anderson 1983; Hartle \& Hu 1979; Fischetti et al. 1979). The basic idea being that anisotropy and/or inhomogeneity would drive gravitational particle creation, which due to back reaction effects would eliminate particle horizons and smooth out the geometry. Recently, Hawking and Hartle (1983) have advocated the Quantum Cosmology approach to actually compute the initial state. All of these approaches necessarily involve events at times $\lesssim 10^{-43} \mathrm{sec}$ and energy densities $\gtrsim m_{p l}^{4}$.

* Anthropic Principle-Some (Carr \& Rees 1979; Barrow \& Tipler 1986) have suggested (or in some cases even advocated) 'explaining' many of the puzzling features of the Universe around us (and in some cases, even the laws of physics!) by arguing that unless they were as they are intelligent life would not have been able to develop and observe them! Hopefully we will not have to resort to such 
an explanation.

The approach of inflation is somewhat different from previous approaches. Inflation (at least from my point-of-view) is based upon well-defined and reasonably well-understood microphysics (albeit, some of it very speculative). That microphysics is:

- Classical Gravity (general relativity), at least as an effective, low energy theory of gravitation

- 'Modern Particle Physics'-grand unification, supersymmetry /supergravity, field theory limit of superstring theories at energy scales $\lesssim m_{p l}$

As I will emphasize, in all viable models of inflation the inflationary period (at least the portion of interest to us) takes place well after the planck epoch, with the energy densities involved being far less than $m_{p l}^{k}$ (although semi-classical quantum gravity effects might have to be included as non-renormalizable terms in the effective Lagrangian). Of course, it could be that a resolution to the cosmological puzzles discussed above involves both 'modern particle physics' and quantum gravitational effects in their full glory (as in a fully ten dimensional quantum theory of strings).

I will not take the time or the space here to review the historical development of our present view of inflation; I refer the interested reader to the interesting paper on this subject by Lindley (1985). It suffices to say that Guth's very influential paper of 1981 initiated the inflation revolution, and that Guth's doomed original model (Guth \& Weinberg 1983; Hawking et al. 1982) was revived by Linde's (1982a) and Albrecht and Steinhardt's (1982) variant, 'new inflation'. I will focus all of my attention on the present status of the 'slow-rollover' model of Linde (1982a) and Albrecht \& Steinhardt (1982).

\section{BASIC MECHANICS OF NEW INFLATION}

Stated in the most general terms, the current view of inflation is that it involves the dynamical evolution of a very weakly-coupled scalar field (hereafter referred to as $\phi$ ) which is, for one reason or another, initially displaced from the minimum of its potential (see Fig. 2). While it is displaced from its mimimum, and is slowly-evolving toward that minimum, its potential energy density drives the rapid (exponential) expansion of the Universe, now known as inflation.

The usual assumptions which are made (often implicitly) in order to analyze inflation are:

- A FRW spacetime with scale factor $R(t)$ and expansion rate

$$
H^{2} \equiv(\dot{R} / R)^{2}=8 \pi \rho / 3 m_{p l}^{2}-k / R^{2}
$$

where the energy density is assumed to be dominated by the stress energy associated with the scalar field (in any case, other forms of stress energy rapidly redshift away during inflation and become irrelevant).

- The scalar field $\phi$ is spatially constant (at least on a scale $\gtrsim H^{-1}$ ) with initial value $\phi_{i} \neq \sigma$, where $V(\sigma)=V^{\prime}(\sigma)=0$, and stress energy tensor

$$
T_{\nu}^{\mu}=\text { diagonal }(-\rho, p, p, p)
$$


Figure 2 - Stated in the most general terms, inflation involves the dynamical evolution of a scalar field which was initially displaced from the minimum of its potential, be that minimum at $\sigma=0$ or $\sigma \neq 0$.


$$
\begin{array}{cc}
\rho=V+\dot{\phi}^{2} / 2 & \left(+\nabla \phi^{2} / 2 R^{2}\right) \\
p=-V+\dot{\phi}^{2} / 2 & \left(-\nabla \phi^{2} / 6 R^{2}\right)
\end{array}
$$

(I have indicated the contribution of the spatial gradient terms for future reference.)

- The semi-classical equation of motion for $\phi$ provides an accurate description of its evolution; more precisely,

$$
\begin{gathered}
\phi(t)=\phi_{c l}(t)+\Delta \phi_{Q M} \\
\ddot{\phi}_{c l}+3 H \dot{\phi}_{c l}+\Gamma \dot{\phi}_{c l}+V^{\prime}\left(\phi_{c l}\right)=0
\end{gathered}
$$

where the quantum fluctuations (characterized by size $\Delta \phi_{Q M} \simeq H / 2 \pi$ ) are assumed to be a small perturbation to the classical trajectory $\phi_{c l}(t)$, and $\Gamma$ is the decay width of the $\phi$ particle. Throughout I use units where $\hbar=k_{B}=c=1$; overdot indicates a derivative with respect to proper time and prime with respect to $\phi$. From this point forward I will drop the subscript ' $\mathrm{cl}$ '. I will return later to these assumptions to discuss how they have been or can be relaxed and/or justified.

The semi-classical evolution of $\phi$ naturally splits into three phases: slowroll; coherent scalar field oscillations; and quantum fluctuations.

(1) Slow-Roll Assuming that the scalar potential is sufficiently flat (the requirement being that $\left.9 H^{2} \gtrsim\left|V^{\prime \prime}\right|\right)$ there will be a period when the motion of $\phi$ is friction-dominated, so that the $\bar{\phi}$ term in Eqn(3b) can be neglected and $\dot{\phi}^{2} \ll V$ can be neglected in Eqn(2b). The equation of motion for $\phi$ becomes

$$
\begin{gathered}
3 H \dot{\phi} \simeq-V^{\prime} \\
H^{2} \simeq 8 \pi V(\phi) / 3 m_{p l}^{2}
\end{gathered}
$$


The growth of the scale factor during the slow-roll is approximately exponential since $H \simeq$ constant, and

$$
\begin{gathered}
R_{f} / R_{i}=\exp \left(\int H d t\right) \equiv \exp (N) \\
N \simeq-3 \int H^{2} d \phi / V^{\prime} \simeq 8 \pi m_{p l}^{-2} \int-V(\phi) d \phi / V^{\prime}(\phi)
\end{gathered}
$$

The exponential growth or inflation occurs during the slow-roll phase.

(2) Coherent Scalar Field Oscillations As $\phi$ approaches $\sigma$ the potential steepens sufficiently (or $V(\phi)$ becomes sufficiently small) so that the motion of $\phi$ is no longer friction-dominated (which occurs when $\left|V^{\prime \prime}\right| \gtrsim 9 H^{2}$ ) and $\phi$ begins to oscillate about the minimum of the potential $(\phi=\sigma)$ on a timescale $\left(\simeq\left|V^{\prime \prime}\right|^{-1 / 2}=m_{\phi}^{-1}\right)$ short compared to the Hubble time. During this phase its oscillatory motion can be time-averaged, and the equation for its evolution becomes

$$
\dot{\rho}_{\phi}+3 H \rho_{\phi}+\Gamma \rho_{\phi}=0
$$

whose solution is $\rho_{\phi} \propto \exp (-\Gamma t) / R^{-3}$-precisely that of an unstable, NR particle species. The coherent field oscillations behave like NR matter (as they should, since they correspond to the zero momentum mode of the field), and decay in a time $\Gamma^{-1}$ (= $\tau_{\phi}$, the lifetime of the $\phi$ particle) due to particle creation by the oscillating $\phi$ field. From the particle point-of-view, the oscillations correspond to a very cold condensate of $\phi$ particles, which then decay. During this phase the scale factor $R(t) \propto t^{2 / 3}$. Assuming that the decay products thermalize quickly (or at least are relativistic) and neglecting any relativistic particles present before inflation as they've been exponentially diluted, the evolution of the energy density in radiation produced by the decay of the coherent field oscillations is governed by

$$
\dot{\rho}_{R}+4 H \rho_{R}=\Gamma \rho_{\phi}
$$

The evolution of $\rho_{\phi}, \rho_{R}$, and the entropy per comoving volume ( $\equiv S \propto R^{3} \rho_{R}^{3 / 4}$ ) are shown in Fig. 3. At early times, $t \ll \Gamma^{-1}, \rho_{r} \propto t^{-1}$ and $S \propto t^{5 / 4}$. The entropy per comoving volume levels off when most of the $\phi$ particles have decayed, at $t \simeq \Gamma^{-1}$, and thereafter the energy density in coherent field oscillations decreases exponentially. The reheating process is essentially complete at this time (save for the possible thermalization of the decay products of the $\phi$ particles) and the temperature is about

$$
T_{R H} \simeq\left(\Gamma m_{p l}\right)^{1 / 2}
$$

Figure 3 summarizes the evolution of $\phi$ and the reheating process. For further discussion see Turner (1983).

(3) Quantum Fluctuations During inflation $\phi$ is on the flat part of its potential and can be treated as an effectively massless scalar field. The spectrum of de Sitter space quantum fluctuations is given by (Bunch \& Davies 1978)

$$
\Delta \phi_{Q M}^{2} \equiv\left|\delta \phi_{k}\right|^{2} k^{3} /(2 \pi)^{3}=H^{2} / 16 \pi^{3}
$$


Figure 3 - The evolution of the energy density in the scalar field $\left(\rho_{\phi}\right)$, in radiation $\left(\rho_{R}\right)$, and of the entropy per comoving volume, $S \propto R^{3} \rho_{R}^{3 / 4}$.

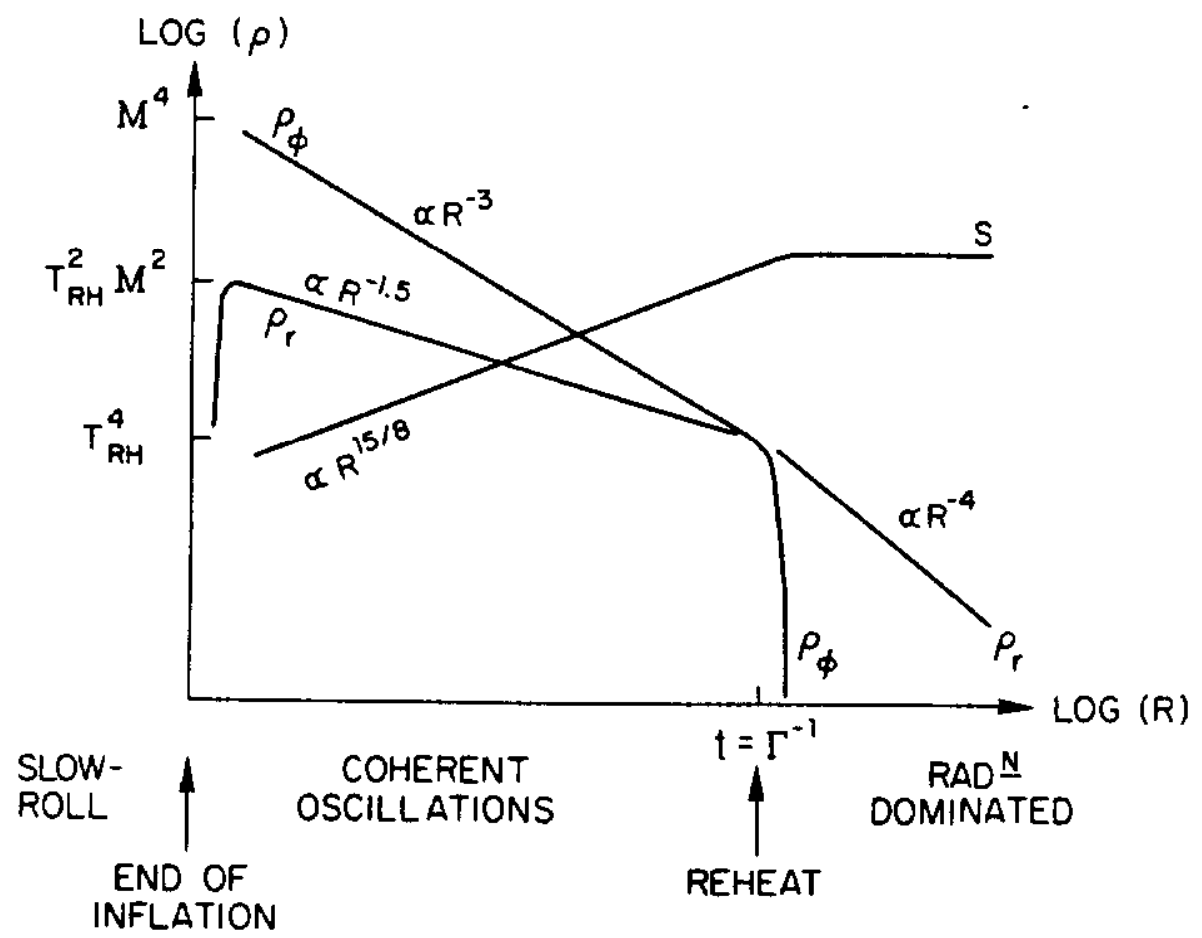

where $\delta \phi_{k}$ is the $k$ - th Fourier component of $\delta \phi$ and $k_{\text {phys }}=k / R(t)$ is the physical wavenumber of the mode with comoving wavenumber $k$. For scales $k_{\text {phye }} \gtrsim H^{-1}$ these perturbations are treated quantum mechanically; as $k_{\text {phys }}$ becomes $\lesssim H^{-1}$, and a given scale crosses outside the 'physics horizon' (or Hubble radius) $\mathrm{H}^{-1}$ the fluctuation on that scale is taken to evolve classically thereafter (the quantum fluctuation is assumed to 'freeze in' as a classical perturbation in the metric). The evolution of the metric perturbations due to the classical fluctuations is straightforward to compute. They give rise to curvature (scalar mode) fluctuations of amplitude (Bardeen et al. 1983; Guth \& Pi 1982; Starobinskii 1982; Hawking 1982)

$$
(\delta \rho / \rho)_{H O R} \simeq k^{3 / 2}\left|\delta_{k}\right| /(2 \pi)^{3 / 2} \simeq\left\{\begin{array}{l}
\left.\left(H^{2} / \pi^{3 / 2} \dot{\phi}\right)\right|_{t_{1}} \\
\left.\left(H^{2} / 10 \pi^{3 / 2} \dot{\phi}\right)\right|_{t_{1}}
\end{array}\right.
$$

when they reenter the horizon. Perturbations which reenter when the Universe is radiation-dominated do so as pressure waves (in the baryon-photon fluid) of the amplitude indicated in Eqn(9a); those which reenter when it is matter-dominated do so as growing mode perturbations with the amplitude indicated in $\operatorname{Eqn}(9 \mathrm{~b})$.

Tensor mode metric (gravitational wave) perturbations also arise and are of dimensionless amplitude (Abbott \& Wise 1984; Starobinskii 1979; Rubakov et al. 1982)

$$
\left.\left.h_{G W}\right|_{H O R} \simeq\left(H / m_{p l}\right)\right|_{t_{1}}
$$


Figure 4- The evolution of the physical wavelength of a given mode. Early during inflation $\lambda_{\text {phya }} \lesssim H^{-1}$ and the scale is inside the physics horizon and fluctuations are treated quantum mechanically. As the mode leaves the physics horizon $\left(\lambda_{\text {phys }} \geq H^{-1}\right)$, the perturbation is assumed to 'freeze in' as a classical metric perturbation.

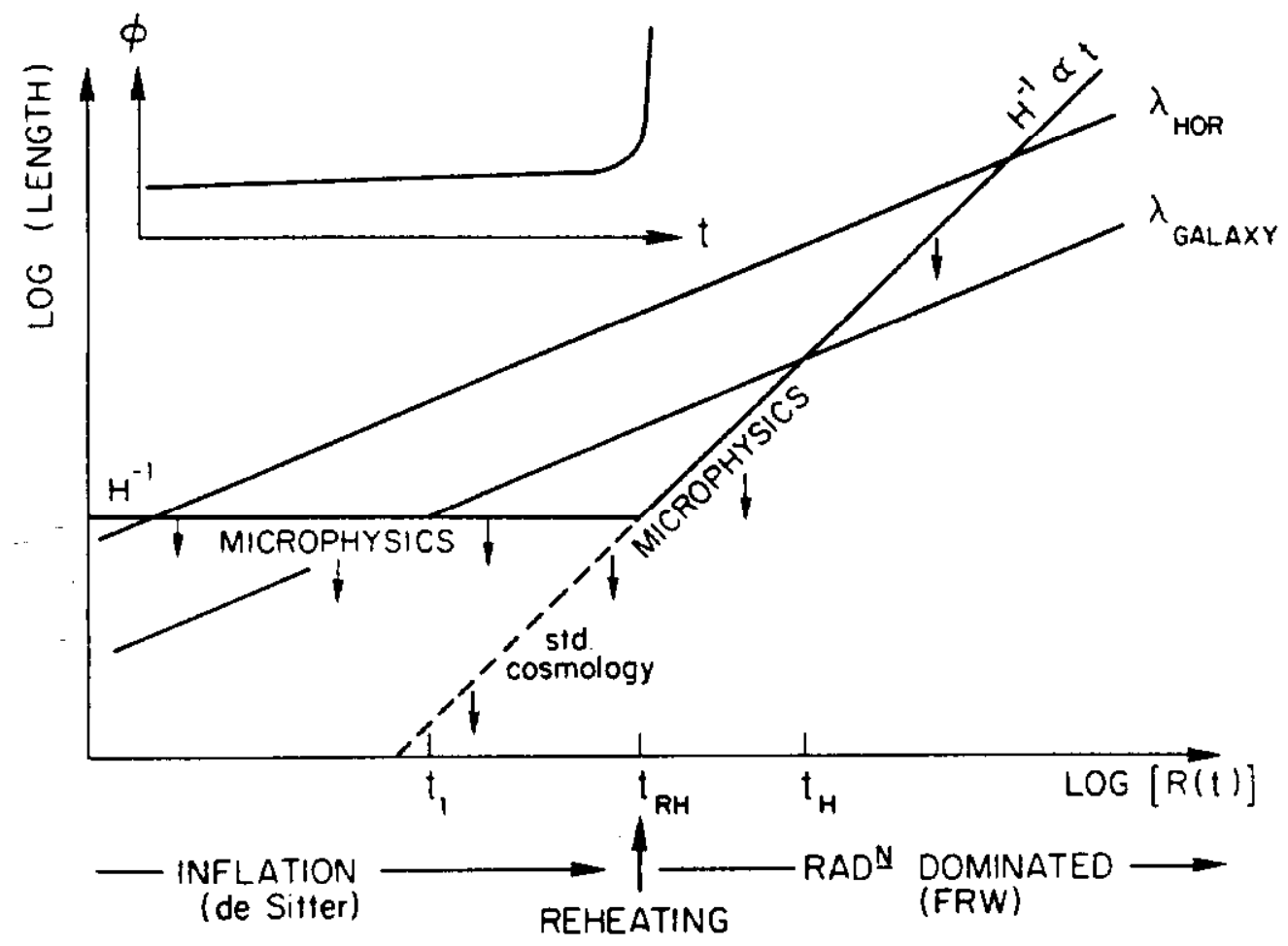

when they reenter the horizon after inflation. In both cases the quantities on the rhs are to be evaluated when the comoving scale of interest crossed outside the Hubble radius $(k / R \simeq H)$, at $t=t_{1}$ (see Fig. 4). Normalizing $R_{\text {today }}=1$, it follows that a given scale $\mathrm{k}(\equiv 2 \pi / \lambda)$ crossed outside the horizon during inflation

$$
N(\lambda) \simeq 45+\ln (\lambda / M p c)+2 \ln \left(M / 10^{14} \mathrm{GeV}\right) / 3+\ln \left(T_{R H} / 10^{10} \mathrm{GeV}\right) / 3
$$

Hubble times (or e-folds) before the end of inflation (see Fig. 4). Thus the scales of astrophysical interest crossed outside the horizon $\mathbf{4 0} \pm 10$ or so Hubble times before the end of inflation. Although $H$ and $\dot{\phi}$ can vary considerably during inflation, over such a small range of Hubble times both $H$ and $H^{2} / \dot{\phi}$ are essentially constant (vary by less than a factor of 2 in all the models I have studied) and so a generic prediction of inflationary models is scale invariant curvature and tensor perturbations. [In models where there are other massless fields, quantum fluctuations of order $H / 2 \pi$ arise in these fields too during inflation and can give rise to isocurvature (often called isothermal) perturbations. A simple example being axion models where the quantum fluctuations in the axion field result in fluctuations in the local axion to entropy density ratio $n_{a} / s$ (see Steinhardt \& Turner 1983; Linde 1985; Seckel \& Turner 1985).] The mechanics of inflation are described in much greater detail in the review by Turner (1986a). 


\section{SUCCESSFULLY IMPLEMENTING INFLATION}

Now that I have discussed the basic mechanics of inflation, how does one build a model which actually leads to a Universe which resembles ours in regions as large as our current Hubble volume? It's as easy as $1,2,3,4,5,6,7,8, \ldots$

(1) Smoothness/Flatness-After inflation there must be smooth/flat regions of the Universe which contain an entropy of at least $10^{88}$. Assuming that $\phi$ was constant in a region of size of order the Hubble radius before inflation, it is straightforward to show that the number of e-folds of inflation required so that the patch contains an entropy of at least $10^{88}$ after reheating is

$$
N \equiv \int H d t \simeq \int-3 H^{2} d \phi / V^{\prime} \gtrsim 53+2 \ln \left(M / 10^{14} \mathrm{GeV}\right) / 3+\ln \left(T_{R H} / 10^{10} \mathrm{GeV}\right) / 3
$$

The number of e-folds required to solve the 'flatness problem' is equal to this plus $\ln \left|1-\Omega_{i}^{-1}\right|$ where $\Omega_{i}$ is the value of $\Omega$ at the beginning of inflation. In general, if a given model inflates at all, it is not difficult to make it inflate enough to solve the smoothness/flatness problems.

(2) Sufficiently Large Smooth Patch-In order that the vacuum energy contribution to the energy density of the Universe dominates the gradient terms $\left((\nabla \phi)^{2}\right), \phi$ must be smooth over a sufficiently large region. [If the gradient term dominates, the stress energy of the scalar field behaves like a fluid with $p=-\rho / 3$ and the scale factor only grows as $R(t) \propto t$, not exponentially.] The condition that $V \gg(\nabla \phi)^{2}$ requires $\phi$ to be smooth over a region of size greater than $\phi_{i} / V^{1 / 2} \simeq\left(\phi_{i} / m_{p l}\right) H^{-1}$.

(3) Validity of Semi-Classical Description-In order that the semi-classical equations of motion be self consistent $\Delta \phi_{Q M} \sim H$ must be much less than $\phi_{c l}$. I will return to this point later-in general it is not a difficulty.

(4) Topological Beastg-One must be careful not to produce any of the dangerous topological beasts such as domain walls, monopoles, etc. after inflation. This can be arranged by having the symmetry breaking stages which result in the formation of such objects occur before or early on during inflation.

(5) Other Unwanted Garbage-As is well-known the ratio of the energy density in nonrelativistic (NR) particles relative to that in relativistic (R) particles grows with time: $\rho_{N R} / \rho_{R} \propto R(t)$. Since our Universe did not become matterdominated until rather recently, $T \simeq 10 \mathrm{eV}$ and $t \simeq 10^{10} \mathrm{sec}$, the ratio of energy density in stable, NR particles (which do not come into thermal equilibrium, or annihilate) to that in $\mathbf{R}$ particles after inflation must be small:

$$
\rho_{N R} / \rho_{R} \lesssim 10^{-19}\left(10^{10} \mathrm{GeV} / T_{R H}\right)
$$

-which is not always an easy thing to do. [Just ask any experimentalist about suppressing something by 19 orders-of-magnitude!] Examples of potentially dangerous forms of NR matter include: gravitinos, weakly-coupled scalar fields which may be put into oscillation after inflation (the so-called Polonyi problem; e.g., see Coughlan et al. 1983), etc. There are even stronger limits on unstable NR particles (particularly 
gravitinos) which follow by considering their effect on primordial nucleosynthesis (Ellis et al. 1984; Khlopov \& Linde 1984; Scherrer \& Turner 1987).

(6) Metric Perturbationo-While the eventual development of structure in the Universe requires density perturbations of the order of $f e w \times 10^{-5}$ or so, the observed isotropy of the CMBR precludes scalar or tensor perturbations of size greater than about $10^{-4}$. Achieving tensor mode perturbations of this size or smaller is not difficult; it only requires that inflation occur at an energy scale $M \simeq V^{1 / 4} \lesssim 10^{-2} m_{p l}$, putting an upper limit on the reheating temperature: $T_{R H} \lesssim 3 \times 10^{16} \mathrm{GeV}$. The scalar perturbations are another matter; thus far, they have posed the most serious obstacle to constructing a successful model of inflation. To achieve curvature fluctuations of amplitude less than about $10^{-4}$ requires a dimensionless parameter in the scalar potential of the order of $10^{-12}$. For example, for a potential of the form $V=\lambda \phi^{4}$, $\lambda$ must be $\lesssim 10^{-13}$; for the potential $V=V_{0}+\alpha \phi^{2}-\beta \phi^{3}+\lambda \phi^{4}, \alpha \lesssim 10^{-15} m_{p l}^{2}$, $\lambda \lesssim 10^{-11}, \beta \lesssim 3 \times 10^{-12} m_{p l}$; for the potential $V=m^{2} \phi^{2}$ (i.e., a non-interacting, massive scalar field), $m^{2} \lesssim 10^{-9} \mathrm{~m}_{p l}^{2}$. Achieving density perturbations of an acceptable amplitude necessitates a very weakly-coupled scalar field; weakly-coupled to all fields in the theory or else radiative corrections would spoil the small coupling put in at tree level. Such a small coupling also implies that inflation takes place at an energy scale much less than the planck scale; typically, $V \simeq \lambda \phi^{4} \simeq 10^{-12} m_{p l}^{4}$ or less.

(7) Sufficient Reheating-In order not to spoil the concordance of the predictions of primordial nucleosynthesis with the observed abundances, the Universe should be radiation-dominated when $t \simeq 0.01 \mathrm{sec}$ and $T \simeq 10 \mathrm{MeV}$ at the very latest, i.e., $T_{R H} \gtrsim 10 \mathrm{MeV}$. This implies that $\Gamma$ must be $\gtrsim 10^{-23} \mathrm{GeV}$-which is not difficult to achieve, even for a very weakly-coupled scalar field. Baryogenesis, however, poses a more formidible challenge. It goes without saying that baryogenesis must follow inflation, as any baryon asymmetry produced before inflation is diluted away by the enormous entropy produced by inflation. If baryogenesis is to proceed in the usual way, $T_{R H}$ must be greater than about a $m_{H} / 10$, where $m_{H}$ is the mass of the superheavy boson whose out-of-equilibrium decays produce the baryon asymmetry (see Kolb \& Turner 1983). In most unified theories the longevity of the proton requires the masses of superheavy bosons whose interactions violate B-conservation to be greater than about $10^{10} \mathrm{GeV}$, thereby requiring $T_{R H} \gtrsim 10^{9} \mathrm{GeV}$ at the very least. Because the scalar field must be very weakly-coupled (to produce density perturbations of an acceptable magnitude) $\Gamma$ tends to be very small, and sufficient reheating is often very difficult to achieve. There is, however, an alternative method which does not require such a high reheating temperature: the direct production of the baryon asymmetry by the decays of the scalar field responsible for inflation $\left(\phi \rightarrow q^{\prime} s\right.$, l's with $\left.\Delta B \neq 0\right)$. In this case the baryon asymmetry produced is

$$
n_{B} / s \simeq \epsilon T_{R H} / m_{\phi}
$$

where $\epsilon$ is the magnitude of the requisite $C, C P$ violation in the decay of the $\phi$ particle. Note that the asymmetry produced only depends upon the ratio of the 
reheating temperature to the mass of the $\phi$ particle, and so it is possible to have a relatively low reheating temperature and still a baryon asymmetry of the required magnitude.

(8) Part of a Unified Model Which Predicts Sensible Particle Physics-In order to avoid having the tail wag the dog so to speak, the scalar field should be part of a unified theory which predicts sensible particle physics.

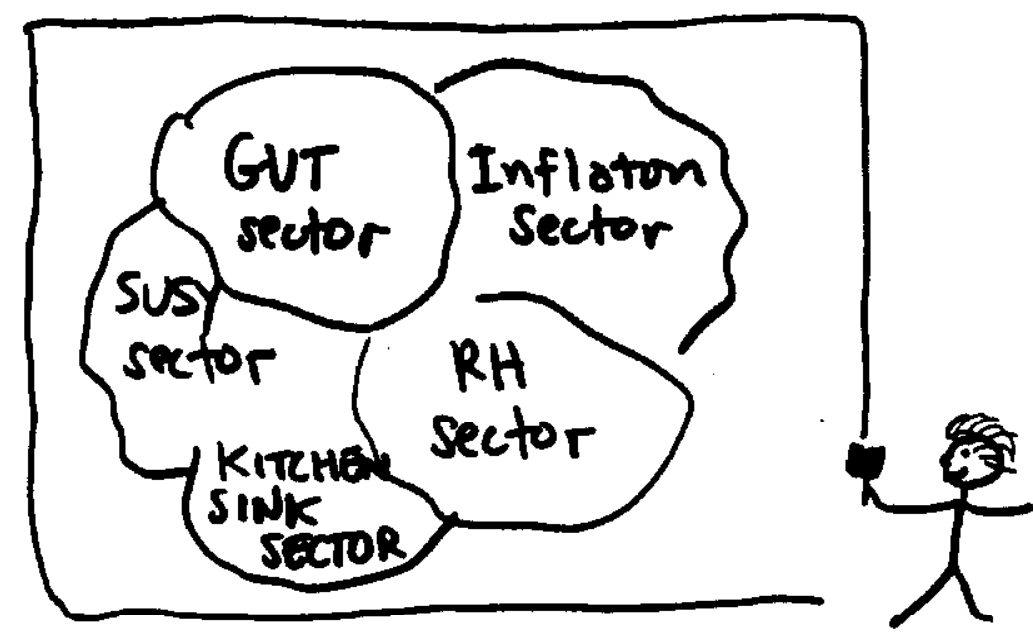

JUST

NB:^PUTTING A BOX. AROUND IT DOES NOT

MAKE IT A'UNIFIED MODE'!!

\section{SPECIFIC MODELS}

While the requirements on a successful model of inflation are straightforward they are not simple to satisfy simultaneously, and the path to a successful model is strewn with the remains of many an attractive model that failed for one reason or another. The toughest challenge has been the constraint imposed by the scalar density perturbations. At present there are no successful models which are so elegant as to be compelling (compelling here, meaning attractive to other than the authors of the model!), although there do exist a number of 'proof of existence' models. I will describe two particularly simple models here.

(1) Shafi-Vilenkin-Pi Model-This model (Shafi \& Vilenkin 1984; Pi 1984) is based upon an $S U_{5}$ nonsupersymmetric GUT (although the gauge group could just as well be $S O_{10}$ or $E_{6}$ ). The scalar field responsible for inflation is a complex Higgs gauge singlet, which in addition to being responsible for inflation, also breaks a PecceiQuinn (PQ) symmetry and effects GUT symmetry breaking by inducing a negative mass squared for the adjoint Higgs representation which then breaks the GUT down to $S U(3) \otimes S U(2) \otimes U(1)$. The part of the Higgs potential which is relevant for inflation is a Coleman-Weinberg type potential

$$
V(\phi)=B \sigma^{4} / 2+B \phi^{4}\left[\ln \left(\phi^{2} / \sigma^{2}\right)-1 / 2\right]
$$


where $\sigma \simeq 10^{18} \mathrm{GeV}$ is the vacuum expectation value of $\phi$ at low temperatures, which breaks PQ symmetry and induces GUT SSB; $B \simeq 10^{-15}$ arises due to radiative corrections from the coupling of $\phi$ to other scalar fields in the theory. Interestingly enough isocurvature axion fluctuations of similar magnitude to the curvature fluctuations also arise in this model (Seckel \& Turner 1985).

(2) Florida SUGR-This model (Holman et al. 1984) is based on an effective low energy supergravity theory with a superpotential of the form, $I+S+G$, where the three pieces of the superpotential are responsible for inflation, supersymmetry breaking and GUT symmetry breaking respectively. The inflation piece of the superpotential takes a particularly simple form

$$
I=\left(\Delta^{2} / M\right)(\phi-M)^{2}
$$

where $\Delta$ is the only adjustable parameter and corresponds to the intermediate scale and $M=m_{p l} /(8 \pi)^{1 / 2} \simeq 2.43 \times 10^{18} \mathrm{GeV}$. The resulting scalar potential for $\phi$ is given by

$$
\begin{gathered}
V_{I}(\phi)=\Delta^{4} \exp \left(\phi^{2} / M^{2}\right)\left[\phi^{6} / M^{6}-4 \phi^{5} / M^{5}+7 \phi^{4} / M^{4}-4 \phi^{3} / M^{3}-\phi^{2} / M^{2}+1\right] \\
\simeq \Delta^{4}\left[1-4 \phi^{3} / M^{3}+6.5 \phi^{4} / M^{4}-8 \phi^{5} / M^{5}+\ldots\right.
\end{gathered}
$$

Achieving density perturbations of an acceptable magnitude requires that $(\Delta / M) \simeq$ $9 \times 10^{-5}$. Note that the coefficient of the $\phi^{4}$ term in $V_{I}$ is dimensionless and for this value of $\Delta$ is about $4 \times 10^{-16}$-the small dimensionless parameter which always arises. Of course, in this model it is directly related to the smallness of the intermediate scale relative to the planck scale, suggesting (or offering hope) that the very small parameter needed for successful inflation is related to fundamental physics. This model reheats to a temperature of about $10^{6} \mathrm{GeV}$ and baryogenesis is effected directly through the decays of the $\phi$ field.

\section{OPEN (or semi-open) QUESTIONS}

* 'Who is $\phi$ ?'-Inflationary models exist in which the scalar field $\phi$ : effects SSB of the GUT (Shafi \& Vilenkin 1984; Pi 1984), effects SSB of SUSY (Ovrut \& Steinhardt 1984a,b), induces Newton's constant (in a Landau-Ginzburg model of induced gravity) (Accetta et al. 1985; Spokoiny 1984), is $\sim \ln \left(r_{X} / r_{X E Q}\right)$ (where $r_{X}$ is the radius of compactified extra dimensions) in theories with extra dimensions which become compactified (Shafi \& Witterich 1983, 1985), is $\propto$ (scalar curvature $)^{1 / 2}$ (Starobinskii 1980; Mijic et al. 1986), is just some 'random' scalar field (Linde 1983), or is merely in the theory to effect inflation (Holman et al. 1984; Nanopoulos 1985). Given the number of different kinds of inflationary scenarios which exist, it seems as though inflation is generic to early Universe microphysics, occurring whenever a weakly-coupled scalar field finds itself displaced from the minimum of its potential. Clearly, a key question at this point is just how 'the inflation sector' of the theory fits into the Big Picture!

* What Determines the Initial Value of $\phi$ ?-One thing is certain, and that is that $\phi$ must be very weakly-coupled, as quantified by its small dimensionless 
coupling constant. Because of this fact, it is almost certain that $\phi$ was not initially in thermal contact with the rest of the Universe and so $\phi_{i}$ is unlikely to be determined by thermal considerations (in the earliest models of new inflation, $\phi_{i}$ was determined by thermal considerations, however these models resulted in density perturbations of an unacceptably large amplitude). At present $\phi_{i}$ must be taken as initial data. Some have argued that $\phi_{i}$ might be determined in an anthropic-like way, as regions of the Universe where $\phi_{i}$ is sufficiently far displaced from equilibrium will undergo inflation and eventually occupy most of the physical volume of the Universe. Perhaps the wavefunction of the Universe approach will shed some light on the initial distribution of the scalar field $\phi$. Or it could be that due to 'as-of-yet unknown dynamics' $\phi$ was indeed in thermal equilibrium at a very early epoch. It goes without saying that it is crucial that $\phi$ be initially displaced from its minimum.

$\star$ Validity of the Semi-Classical Equations of Motion for $\phi-$ While it may seem perfectly plausible that $\phi$ evolves according to its semi-classical equations of motion, the validity of this assumption has troubled inflationists from the 'dawn of new inflation'. While a full quantum field theory treatment of inflation is very difficult and has not been effected, a number of specific issues have been addressed. Several authors have studied the role of inhomogeneities in $\phi$, and have found that for the very weakly-coupled fields one is dealing with, mode coupling is not important and the individual modes are quickly smoothed by the exponential expansion of their physical wavelengths (Albrecht \& Brandenberger 1985; Albrecht et al. 1985). I already mentioned the necessity of having $\phi$ smooth over a sufficiently large region so that the gradient terms in the stress energy do not dominate.

The effect of quantum fluctuations on the evolution of $\phi$ has been studied in some detail by Guth \& Pi (1985), Fischler et al. (1985), Linde (1982b), and Vilenkin \& Ford (1982). The basic conclusion that one draws from the work of these authors is that the use of the semi-classical equations of motion is valid so long as $\phi_{c l} \gg$ $\Delta \phi_{Q M} \simeq N^{1 / 2} H / 2 \pi$, which is almost always satisfied for the very flat potentials of interest to inflationists (at least for the last 50 or so e-folds which affect our present Hubble volume). (More precisely, the semi-classical change in $\phi$ in a Hubble time, $\Delta \phi_{\text {Hubble }} \simeq-V^{\prime} / 3 H^{2} \simeq-V^{\prime} m_{p l}^{2} /(8 \pi V)$, should be much greater than the increase in $\left\langle\phi^{2}>_{Q M}^{1 / 2}\right.$, which is of order $H / 2 \pi$, due to the addition of an another quantum mode; see Bardeen et al. 1983.] At present the validity of the semi-classical equations of motion seems to reasonably well established.

* No Hair Conjectures-While inflation has been touted from the very beginning as making the present state of the Universe insensitive to the initial spacetime geometry, not much has been done to justify this claim until very recently. As I mentioned earlier, inflation is nearly always analyzed in the context of a flat, FRW cosmological model, making such a claim somewhat dubious. However, it has now been shown that all of the homogeneous models (with the exception of the highlyclosed models) undergo inflation, isotropize and remain isotropic to the present epoch providing that the model would have inflated the requisite 60 or so e-folds in the 
absence of anisotropy (Turner \& Widrow 1986; Jensen \& Stein-Schabes 1986a).

The proof of this result involves three parts. First, Wald (1983) demonstrated that all homogeneous models with a positive cosmological term asymptotically approach deSitter (less the aforementioned highly-closed models which recollapse before the cosmological term becomes relevant). Wald's result follows because all forms of 'anisotropy energy density' decrease with increasing proper volume element, whereas the cosmological term remains constant, and so eventually triumphs. Of course, inflationary models do not in the strictest sense, have a cosmological term, rather they have a positive vacuum energy as long as the scalar field is displaced from the minimum of its potential. Thus the dynamics of the scalar field comes into play: does $\phi$ stay displaced from the minimum of its potential long enough so that the vacuum energy comes to dominate? Due to the presence of anisotropy the expansion rate is greater than if there were only vacuum energy density, and so the friction felt by $\phi$ as it trys to roll (the $3 H \dot{\phi}$ term) is greater and it takes $\phi$ longer to evolve to its minimum than without anisotropy. For this reason the Universe does become vacuum dominated before the vacuum energy disappears, and in fact the Universe inflates slightly longer in the presence of anisotropy (one or two e-folds) (Steigman \& Turner 1983). Finally, is the anisotropy reduced sufficiently so that the Universe today is still nearly isotropic? As it turns out, the requisite 60 or so e-folds needed to solve the other conundrums reduces the growing modes of anisotropy sufficiently to render them small today.

Allowing for inhomogeneous initial spacetimes makes matters much more difficult. Jensen and Stein-Schabes (1986b) and Starobinskii (1983) have proven the analogue of Wald's theorem for spacetimes which are negatively-curved. Jensen and Stein-Schabes (1986b) have gone on to conjecture that spacetimes which have sufficiently large regions of negative curvature will undergo inflation, resulting in a Universe today which although not globally homogeneous, at least contains smooth volumes as large as our current Hubble volume.

Does this improve the situation that Collins and Hawking discussed in 1973? While the work of Jensen and Stein-Schabes (1986) seems to indicate that many inhomogeneous spacetimes undergo inflation and even leads one to speculate that the measure of the set of initial spacetimes which eventually inflate is non-zero, it is not possible to draw a definite conclusion without first defining a measure on the space of initial data. In fact, as Penrose (1979) pointed out there is at least one way of defining the measure such that this is not the case. Consider the set of all Cauchy data at the present epoch; intuitively it is clear that those spacetime slices which are highly irregular are the rule, and those which are smooth in regions much larger than our current Hubble volume are the exception. Defining the measure today, it seems very reasonable that the smooth spacetime slices are a set of measure zero. Now evolve the spacetimes back to some initial epoch (for example $t=10^{-43} \mathrm{sec}$ ). Using the seemingly very reasonable measure defined today and the mapping back to 'initial' spacetimes, one could argue that the set of initial data which inflate is still of measure zero. While I believe that this argument is technically correct, I also believe that it is silly. First, 
upon close examination of all of those initial spacetimes which led to spacetimes today without smooth regions as large as our present Hubble volume, one would presumably find that the scalar field in most would be very close to the minimum of its potential (in order that they not inflate)-not a very generic initial condition. Secondly, if one adopts the point-of-view of an evolving Universe which has an 'initial epoch' (and not everyone does), then there is a preferred epoch at which one would define a measurethe 'initial epoch,' and at that epoch I believe any reasonably defined measure would lead to the set of initial spacetimes which inflate being of non-zero measure.

Although it is not possible yet to claim rigorously that inflation has resolved the problem of the seemingly special initial data required to reproduce the Universe we see today (at least within our Hubble volume), I think that any fairminded person would admit that it has improved the situation dramatically. Extrapolating from the solid results that exist, it seems to me that starting with a general inhomogeneous spacetime, there will exist regions which undergo inflation and which today are much larger than our present Hubble volume, thereby accounting for the smooth region we find ourselves in. From a more global perspective, one might expect that on scales $\gg H^{-1}$ the Universe would be highly irregular.

* The Present Vanishingly Small Value of the Cosmological Constant-Inflation has shed no light on this difficult and very fundamental puzzle (nor has anything else for that matter!). In fact, since inflation runs on vacuum energy so to speak, the fate of inflation hinges upon the resolution of this puzzle. For example, suppose there were a grand principle that dictated that the vacuum energy of the Universe is always zero, or that there were an axion-like mechanism which operated and ensured that any cosmological constant rapidly relaxed to zero; either would be a disaster to inflation, shorting out its source of power-vacuum energy. [Another possiblity which has received a great deal of attention recently is the possibility that deSitter space might be quantum mechanically unstable-of course, if its lifetime were at least 60 some e-folds that would not necessarily adversely affect inflation (Starobinskii 1980; Myhrvad 1983; Mottola 1985; Parker 1983; Ford 1985; Anderson 1985; Traschen \& Hill 1986).]

\section{INFLATION CONFRONTS OBSERVATION}

No matter how appealing a theory may be, it must meet and pass the test of experimental verification. Experiment and/or observation is the final arbiter. One of the few blemishes on early Universe physics is the lack, thus far, of experimental/observational tests of the many beautiful and exciting predictions. That situation is beginning to change as the field starts to mature. Inflation is one of the early Universe theories which is becoming amenable to verification or falsification. Inflation makes the following very definite predictions (postdictions?):

$\left.H^{-1}\right)$

- $\Omega=1.0$ (more precisely, $R_{c u r v}=R(t)|k|^{-1 / 2}=H^{-1} /|\Omega-1|^{1 / 2} \gg$

- Harrison-Zel'dovich spectrum of constant curvature perturbations (and possibly isocurvature perturbations as well) and tensor mode gravitational wave mode 
perturbations

The prediction of $\Omega=1.0$ together with the primordial nucleosynthesis constraint on the baryonic contribution, $0.014 \lesssim \Omega_{B} h^{2} \lesssim 0.035 \lesssim 0.15$ (Yang et al. 1984), suggests that most of the matter in the Universe must be nonbaryonic. The simplest and most plausible possibility is that it exists in the form of relic WMMs (Weakly-Interacting Massive Particles, e.g., axions, photinos, neutrinos; for a review, see Turner 1986b). Going a step further, these two original predictions then lead to testable consequences:

$\star H_{0} t_{0}=2 / 3$ (providing that the bulk of the matter in the Universe today is in the form of NR particles)-The observational data on both $H_{0}$ and $t_{0}$ are far from being definitive: $H_{0} \simeq 40-100 \mathrm{kmsec}^{-1} \mathrm{Mpc} \mathrm{se}^{-1}$ and $t_{0} \simeq 12-20 \mathrm{Gyr}$, implying only that $H_{0} t_{0} \simeq 0.5-2.0$.

$\star \Omega=1.0$-All of the dynamical observations suggest that the fraction of critical density contributed by matter which is clumped on scales $\lesssim 10-30 \mathrm{Mpc}$ is only about: $\Omega_{\lesssim 30} \simeq 0.2 \pm 0.1( \pm 0.1$ is not meant to be a formal error estimate, but indicates the spread in the observations) (see the recent review by Trimble 1987). If inflation is not to be falsified, that leaves but two options: (1) the observations are somehow misleading or wrong; or (2) there exists a component of energy density which is smoothly distributed on scales $\lesssim 10-30 M p c$ (and therefore would not be reflected in the dynamical determinations). Candidates for the smooth component include: relic, light neutrinos, which by virtue of the large length scale $\left(\lambda_{\nu} \simeq 13 h^{-2} \mathrm{Mpc}\right)$ on which neutrino perturbations are damped by freestreaming, would likely still be smooth on these scales; relic relativistic particles produced by the recent decay of an unstable WIMP species (Turner et al. 1984; Dicus et al. 1977; Olive et al. 1985); a relic cosmological term (Turner et al. 1984; Peebles 1984); 'failed galaxies,' referring to a population of galaxies which have the same mix of dark matter to baryons, but are more smoothly distributed and are too faint to observe (at least.thus far) (Kaiser 1984, 1986; Bardeen et al. 1986); a relic population of light strings-either fast moving non-intercommuting strings or a tangled network of non-Abelian strings (Vilenkin 1984). All of these smooth component scenarios have testable consequences (Charlton \& Turner 1987)-their predictions for $H_{0} t_{0}$ differ from 2/3; the growth of perturbations is different; the evolution of the cosmic scale factor $R(t)$ is different from the matter-dominated model and various kinematic tests (magnitude-redshift, angular size-redshift, lookback time-redshift, proper volume element-redshift, etc.) can in principle differentiate between them.

* Microwave Fluctuationo-Both the scalar and tensor metric perturbations, cf. Eqns(9.10), lead to fluctuations in the CMBR on large angular scales $\left(\gg 1^{\circ}\right)$. On such large scales causal processes (such as reionization) cannot have erased the primordial fluctuations, and so if ever present, they must still be there. The scalar perturbations (if they have anything to do with structure formation) must be of amplitude $\gtrsim f e w \times 10^{-6}$, which is within a factor of 10 or less of the current upper limits on these scales. 
* Two Detailed Stories of Structure Formation-The simplest possibility, namely that the most of the mass density is in relic WIMPs $\left(\Omega_{W I M P}=1.0-\Omega_{B} \simeq 0.9\right)$ leads to two very detailed scenarios of structure formation: hot dark matter (the case where the dark matter is neutrinos) and cold dark matter (essentially any other WIMP as dark matter). At present, the numerical simulations of these scenarios are sufficiently definite that it is possible to falsify them-and in fact, both of these simplest scenarios have difficulties (see the recent review by White 1986). In the hot dark matter case it is forming galaxies early enough. The large-scale structure which evolves in this case (voids, superclusters, froth) qualitatively agrees with what is observed; however, in order to get agreement with the galaxy-galaxy correlation function, galaxies must form very recently (redshifts $\lesssim 1$ ) in contradiction to all the galaxies (redshifts as large as 3.2) and QSO's (redshifts as large as 4.0) which are seen at redshifts $\gtrsim 1$.

With cold dark matter the simulations can nicely seproduce galaxy clustering, most of the observed properties of galaxies (masses and densities, rotation curves, etc) (Blumenthal et al. 1984; Davis et al. 1985). However the simulations do not seem to be able to produce sufficient large-scale structure. In particular, they fail to account for the amplitude of the cluster-cluster correlation function (by a factor of about 3), large amplitude, large-scale peculiar velocities, and voids. [In fairness I should mention that our knowledge of large-scale structure of the Universe is still very fragmentary, with the first moderate sized $\left(\sim 10^{4}\right)$, 3-dimensional surveys having just recently been completed.] In order to account for $\Omega=1.0$, galaxy formation must be biased (i.e., only density-averaged peaks greater than some threshold, typically $2-3 \sigma$, are assumed to evolve into galaxies which we see today, the more typical $1 \sigma$ peaks resulting in 'failed galaxies' for some reason or another; see Bardeen et al. 1986).

(The situation with respect to large scale structure is becoming more interesting every moment. Several groups have now reported large-amplitude (600 $\left.1000 \mathrm{kmsec}^{-1}\right)$ peculiar velocities on large scales $\left(\sim 50 \mathrm{~h}^{-1} \mathrm{Mpc}\right.$ ) (Burstein et al. 1986; Collins et al. 1986). Such large peculiar velocities are very difficult, if not impossible, to reconcile with either hot or cold dark matter (or even smooth component models) and the Zel'dovich spectrum (Vittorio \& Turner 1987). If these data hold up they may pose an almost insurmountable obstacle to any scenario with the Zel'dovich spectrum of density perturbations. The frothy structure observed in the galaxy distribution by de Lapparent et al. (1986), galaxies distributed on the surfaces on large ( $\left.\sim 30 h^{-1} M p c\right)$, empty bubbles, although somewhat more qualitative, also seems difficult to reconcile with cold dark matter.]

There are a number of observations/experiments which can and will be done in the next few years and which should really put the inflationary scenario to the test. They include improved sensitivity measurements of the CMBR anisotropy. The microwave background anisotropies predicted in the hot dark matter scenario are very close to the observational upper limits on angular scales of both 5 or so arcminutes and $\gtrsim$ few degrees (Vittorio \& Silk 1984; Bond \& Efstathiou 1984). With 
cold dark matter, the predictions are a factor of $3-10$ away from the observational limits (for the isocurvature spectrum, the quadrupole upper limit may actually rule out this possibility; see, Efstathiou \& Bond 1986). An improvement in sensitivity to microwave anisotropies of the order of 3-10 could either begin to confirm one of the scenarios or rule them both out, and is definitely within the realm of experimental reality (Wilkinson 1986 ).

The relic WIMP hypothesis for the dark matter can also be tested. While it was once almost universally believed that all WIMP dark matter candidates were, in spite of their large abundance, essentially impossible to detect because of the feebleness of their interactions, a number of clever ideas have recently been suggested (and are being experimentally implemented) for detecting axions (Sikivie 1983), photinos, sneutrinos, heavy neutrinos, etc (Goodman \& Witten 1985). Results and/or limits will be forth coming soon. With the coming online of the Tevatron at Fermilab, the SLC at SLAC, and hopefully the SSC it is possible that one of the candidates may be directly produced in the lab. Experiments to detect neutrino masses in the eV mass range also continue.

A geometric measurement of the curvature of the Universe (which uses the dependence of the comoving volume element as a function of redshift) has recently been made by Loh and Spillar (1986). Their preliminary results indicate $\Omega=0.9_{-0.5}^{+0.7}$ ( $95 \%$ confidence) (for a matter-dominated model). This technique appears to have great cosmological leverage and looks very promising-far more promising than the traditional approach of determining the density of the Universe through the deceleration parameter $q_{0}$.

Another area with great potential for improvement is $3 d$ surveys of the distribution of galaxies. The largest redshift surveys at present contain only a few 1000 galaxies, yet have been very tantalizing, indicating evidence of voids and froth-like structure to the galaxy distribution (de Lapparent et al. 1986). The large, automated surveys which are likely to be done in the next decade could very well lead to a quantum leap in our understanding of the large scale features of the Universe and help to provide hints as to how they evolved.

The peculiar velocity field of the Universe is potentially a very valuable and direct probe of the the density field of the Universe:

$$
\begin{gathered}
\left|\delta v_{k}\right|=\left|\dot{\delta}_{k} / k\right| \quad\left(=(\lambda H / 2 \pi) \delta_{k} \text { for } \Omega=1\right) \\
(\delta v / c)_{\lambda} \simeq\left(\lambda / 10^{4} h^{-1} M p c\right)(\delta \rho / \rho)_{\lambda}
\end{gathered}
$$

where $\delta_{k}$ and $\delta v_{k}$ are the $k-t h$ Fourier components of $\delta \rho / \rho$ and $\delta v / c$ respectively. The very recent measurements which indicate large amplitude peculiar velocities on scales of $\sim 50 h^{-1} M p c$ are surprising in that they indicate substantial power on these scales, and are problematic to almost every scenario of structure formation. Should they be confirmed they will provide a very acute test of structure formation in inflationary models. 
Of course, theorists are very accommodating and have already started suggesting alternatives to the simplest scenarios for structure formation. As I mentioned earlier, scenarios with a smooth component to the energy density have been put forward to solve the $\Omega$ problem. Cosmic strings present a radically different approach to structure formation with their non-gaussian spectrum of density fluctuations. [It is interesting to note that cosmic strings of the right 'weight' ( $G \mu \simeq 10^{-6}$ or so, where $\mu$ is the string tension) seem to be somewhat incompatible with inflation, as they must necessarily be produced after inflation and require reheating to a temperature $\gtrsim \mu^{1 / 2} \simeq 10^{16} \mathrm{GeV}$ which seems difficult.] Somewhat immodestly I mention a proposal Silk and I recently made: 'double inflation' (Silk \& Turner 1987; Turner et al. 1987). While the Harrison-Zel'dovich spectrum is a beautiful prediction both because of its geometric simplicity and its definiteness, it may well be in conflict with observation because it does not seem to allow enough power on large scales to account for the recent observations of froth and large amplitude peculiar velocities. In the variant we have proposed there are two (or more) episodes of inflation, with the final episode lasting only about 40 e-folds or so, so that the amplitudes of perturbations on large scales are set by the first episode and those on small scales by the second episode. This enables one to have very large amplitude perturbations on small scales (of order $10^{-1}$ ) and larger than usual amplitude perturbations on large scales (nearly saturating the large scale microwave limits), thereby providing enough power for the large scale structure which the recent redshift surveys and peculiar velocity measurements indicate. The large amplitude perturbations on small scales allow for very early galaxy formation (and reionization of the Universe, thereby erasing the CMBR fluctuations on small angular scales). If the second episode of inflation proceeds via the nucleation of bubbles, they might directly explain the froth-like structure recently reported by de Lapparent (1986).

\section{EPILOGUE}

Despite the absence of a compelling model which successfully implements the inflationary paradigm, inflation remains a very attractive means of accounting for a number of very fundamental cosmological facts by microphysics that we have some understanding of: namely, scalar field dynamics at sub-planck energies. The lack of a compelling model at present must be viewed in the light of the fact that at present we have no compelling, detailed model for the 'Theory of Everything' and the fact that despite vigorous scrutiny there has yet to be a No-Go Theorem for inflation unearthed. It is my belief that the undoing of inflation (if it should come) will involve observations and not theory. At the very least The Inflationary Paradigm is still worthy of further consideration-and I hope that I have convinced you of that fact!

Due to space/time limitations my review of inflation has necessarily been incomplete, for which I apologize. I refer the interested reader to the more complete reviews by Linde (1984); by Abbott \& $\mathrm{Pi}$ (1986); and by Steinhardt (1984); by Brandenberger (1985); and by myself (Turner 1986a). My prescription for successfully implementing inflation borrows heavily from the paper written by Steinhardt and my- 
self (Steinhardt \& Turner 1984). This work was supported in part by the DoE (at Chicago) and by my Alfred P. Sloan Fellowship.

\section{REFERENCES}

Abbott, L. \& Pi, S.-Y. (1986). The Inflationary Universe. Singapore: World Scientific. Abbott, L. \& Wise, M.B. (1984). Nucl. Phys., B244, 541.

Accetta, F. et al. (1985). Phys. Rev., D31, 3046.

Albrecht, A. \& Brandenberger, R. (1985). Phys. Rev., D31, 1225.

Albrecht, A. et al. (1985). Phys. Rev., D32, 1280.

Albrecht, A. \& Steinhardt, P.J. (1982). Phys. Rev. Lett., 48, 1220.

Albrecht, A. \& Turok, N. (1985). Phys. Rev. Lett., 54, 1868.

Anderson, P. (1983). Phys. Rev., D28, 271; D29, 615.

Anderson, P. (1985). Phys. Rev., D32, 1302.

Bardeen, J.M. (1980). Phys. Rev., D22, 1882.

Bardeen, J.M., et al. (1983). Phys. Rev., D28, 679.

Bardeen, J.M., et al. (1986). Astrophys. J., 304, 15.

Barrow, J.D. \& Tipler, F. (1986). The Anthropic Cosmological Principle. Oxford: Oxford University Press.

Blumenthal, G., et al. (1984). Nature, 311, 517.

Boesgaard, A. \& Steigman, G. (1985). Ann. Rev. Astron. Astrophys., 23, 319.

Bond, J.R. \& Efstathiou, G. (1984). Astrophys. J., 285, L44.

Brandenberger, R. (1985). Rev. Mod. Phys., 57, 1.

Bunch, T. \& Davies, P.C.W. (1978). Proc. Roy. Soc. London, A360, 117.

Burstein, D., et al. (1986). Astrophys. J., in press.

Carr, B.J. \& Rees, M.J. (1979). Nature, $278,605$.

Charlton, J. \& Turner, M.S. (1987). Astrophys. J., in press.

Collins, C.A., et al. (1986). Nature, 320, 506.

Collins, C.B. \& Hawking, S.W. (1983). Astrophys. J., 180, 317.

Coughlan, G. et al. (1983). Phys. Lett., 131B, 54.

Davis, M., et al. (1985). Astrophys. J., 292, 371.

deLapparent, V., et al. (1986). Astrophys. J., $302, \mathrm{~L} 1$.

DeWitt, B. (1953). Phys. Rev., 90, 357.

Efstathiou, G. \& Bond, J.R. (1986). Mon. Not. r. Astron. Soc., 218, 103.

Fischler, W. et al. (1985). Nucl. Phys., B259, 730.

Dicke, R.H. \& Peebles, P.J.E. (1979). In General Relativity: An Einstein Centenary Survey, eds. S. Hawking \& W. Israel. Cambridge: Cambridge University Press.

Dicus, D.A., et al. (1977). Phys. Rev. Lett., 39, 168.

Ellis, J.E., et al. (1984). Phys. Lett., 145B, 181.

Efstathiou, G. \& Silk, J. (1983). Fund. Cosmic Phys., 9, 1.

Fischetti, M.V., et al. (1979). Phys. Rev., D20, 1757.

Ford, L. (1985). Phys. Rev., D31, 710.

Goodman, M. \& Witten, E. (1985). Phys. Rev., D31, 3059.

Guth, A. (1981). Phys. Rev., D23, 347.

Guth, A. \& Pi, S.-Y. (1982). Phys. Rev. Lett., 49, 1110.

Guth, A. \& Pi, S.-Y. (1985). Phys. Rev., D32, 1899.

Guth, A. \& Weinberg, E. (1983). Nucl. Phys., B212, 321.

Hartle, J.M. \& Hawking, S.W. (1983). Phys. Rev., D28, 2960. 
Hartle, J.M. \& Hu, B.-L. (1979). Phys. Rev., D20, 1772.

Hawking, S.W. (1982). Phys. Lett., 115B, 295.

Hawking, S.W., et al. (1982). Phys. Rev., D26, 2681.

Holman, R., et al. (1984). Phys. Lett., 137B, 343.

Kaiser, N. (1983). Astrophys. J., 273, L17.

Kaiser, N. (1986). In Inner Space /Outer Space, eds. E.W. Kolb, et al. Chicago: University of Chicago Press.

Khlopov, M.Yu. \& Linde, A.D. (1984). Phys. Lett., 138B, 265.

Kolb, E.W. \& Turner, M.S. (1983). Ann. Rev. Nucl. Part. Sci., 33, 645.

Jensen, L. \& Stein-Schabes, J. (1986a). Phys. Rev., D94, 931.

Jensen, L. \& Stein-Schabes, J. (1986b). Phys, Rev., in press.

Linde, A.D. (1982a). Phys. Lett., 108B, 389.

Linde, A.D. (1982b). Phys. Lett., 116B, 335.

Linde, A.D. (1983). Phys. Lett., 129B, 177.

Linde, A.D. (1984). Rep. Prog. Phys., 47, 925.

Linde, A.D. (1985). Phys. Lett., 158B, 375.

Lindley, D. (1985). Fermilab preprint (unpublished).

Loh, E. \& Spillar, E. (1986). Astrophys. J., 307, L1; Phys. Rev. Lett., 57, 2865.

Matzner, R. \& Misner, C.W. (1972). Astrophys. J., 171, 415.

Mijic, M.B., et al. (1986). Phys. Rev., D34, 2934.

Misner, C.W. (1968). Astrophys. J., 151, 431.

Misner, C.W. (1972). In Magic Without Magic, ed. J. Klauder. San Francisco: Freeman.

Mottola, E. (1985). Phys. Rev., D31, 754; D33, 2136.

Myhrvad, N. (1983). Phys. Lett., 132B, 308.

Nanopoulos, D.V. (1985). Comments on Astrophys., X, 219.

Olive, K., et al. (1985). Astrophys. J., 292, 1.

Ovrut, B. \& Steinhardt, P.J. (1984a). Phys. Rev. Lett., 53, 732.

Ovrut, B. \& Steinhardt, P.J. (1984b). Phys. Lett., 147B, 263.

Parker, L. (1976). Nature, 261, 20.

Parker, L. (1983). Phys. Rev. Lett., 50, 1009.

Peebles, P.J.E. (1984). Astrophys. J., 284, 439.

Penrose, R. (1979). In General Relativity: An Einstein Centenary Survey, eds. S.W.

Hawking \& W. Israel. Cambridge: Cambridge University Press.

Peterson, J., et al. (1985). Phys. Rev. Lett., 55, 332.

Pi, S.-Y. (1984). Phys. Rev. Lett., 52, 1725.

Preskill, J. (1984). Ann. Rev. Nucl. Part. Sci., 34, 461.

Rubakov, V.A., et al. (1982). Phys. Lett., 115B, 189.

Seckel, D. \& Turner, M.S. (1985). Phys. Rev., D32, 3178.

Scherrer, R.J. \& Turner, M.S. (1987). Astrophys. J., in press.

Shafi, Q. \& Vilenkin, A. (1984). Phys. Rev. Lett., 52, 691.

Shafi, Q. \& Wetterich, C. (1983). Phys. Lett., 129B, 387.

Shafi, Q. \& Wetterich, C. (1985). Phys. Lett., 152B, 51.

Sikivie, P. (1983). Phys. Rev. Lett., 51, 1415.

Silk, J. \& Turner, M.S. (1987). Phys. Rev., D35, in press.

Smoot, G.F., et al. (1985). Astrophys. J., 291, L23.

Spokoiny, B.L. (1984). Phys. Lett., 147B, 39.

Starobinskii, A.A. (1979). JETP Lett., 30, 682.

Starobinskii, A.A. (1980). Phys. Lett., 91B, 99. 
Starobinskii, A.A. (1982). Phys. Lett., 117B, 175.

Starobinskii, A.A. (1983). JETP Lett., 37, 66.

Steigman, G. \& Turner, M.S. (1983). Phys. Lett., 128B, 295.

Steinhardt, P.J. (1984). Comments Nucl. Part. Phys., 12, 273.

Steinhardt, P.J. \& Turner, M.S. (1983). Phys. Lett., 129B, 51.

Steinhardt, P.J. \& Turner, M.S. (1984). Phys. Rev., D29, 2162.

Traschen, J. \& Hill, C.T. (1986). Phys. Rev., D33, 3519.

Trimble, V. (1987). Ann. Rev. Astron. Astrophys., 25, in press.

Turok, N. (1985). Phys. Rev. Lett., 5 5, 1801.

Turner, M.S. (1983). Phys. Rev., D28, 1243.

Turner, M.S. (1986a). In Fundamental Physics and Cosmology, eds. J. Audouze \& J. Tran Thanh Van. Gif-sur-Yvette: Editions Frontieres; and in The Architecture of Fundamental Interactions at Short Distances, eds. P. Ramond \& R. Stora. Amsterdam: North-Holland.

Turner, M.S. (1986b). In Dark Matter in the Universe, eds. J. Kormendy \& J. Knapp. Dordrecht: Reidel.

Turner, M.S., et al. (1984). Phys. Rev. Lett., 52, 2090.

Turner, M.S., et al. (1987). Astrophys. J., in press.

Turner, M.S. \& Widrow, L. (1986). Phys. Rev. Lett., 57, 2237.

Vilenkin, A. (1984). Phys. Rev. Lett., 53, 1016.

Vilenkin, A. (1985). Phys. Rep., 121, 263.

Vilenkin, A. \& Ford, L. (1982). Phys. Rev., D26, 1231.

Vittorio, N. \& Silk, J. (1984). Astrophys. J., 285, L39.

Vittorio, N. \& Turner, M.S. (1987). Astrophys. J., in press.

Wald, R.M. (1983). Phys. Rev., D28, 2118.

White, S.D.M. (1986). In Inner Space /Outer Space, eds. E.W. Kolb, et al. Chicago: University of Chicago Press.

Wilkinson, D.T. (1986). In Inner Space /Outer Space, eds. E.W. Kolb, et al. Chicago: University of Chicago Press.

Yang, J., et al. (1984). Astrophys. J., 281, 493.

Zel'dovich, Ya.B. (1970). JETP Lett., 12, 307. 